\title{
处处振荡的达布函数
}

\author{
王 祖 機 \\ (浙江师范学院)
}

Marcus 在文献 [1] 中证明了下述定理.

定理 假设 $f(x)$ 是区间 $I$ 上的连续函数。它在 $I$ 的某一稠密集上点点取到极大值,那么对 于每一个区间 $J \subseteq 1$, 或是存在区间 $K \subseteq J$, 使 $f(x)$ 在 $K$ 上是常数; 或是存在关于 $f(J)=\{y$; $f(x)=y, x \in J\}$ 的第一纲集 $A(J)$, 使对每一个 $t \in f(J)-A(J)$, 集 $J \cap\{x ; f(x)=t\}$ 是不 可数集.

Marcus 又提出了两个问题:

（1）在上述定理中是否可将连续函数改为达布函数?

（2）是否存在这样一个处处可微函数，它在定义区间的一个椆密集上点点取到真正极大 值?

问题 (2) 的回答早已知道 ${ }^{[2]}$. 本文对问题 (1) 作了否定回答, 这就是本文定理 1 ; 而本文 定理 2 说明, 也存在一类非连续的达布函数能满足问题 (1) 的要求.

另外，Дринфель ${ }^{[3]}$ 曾化了较长的篇幅构造了一个处处间断的达布函数，下面定理 1 的 证明给出了一个构造这种函数的简便方法.

定理 1 在区间 $I=[0,1]$ 上存在着达布函数 $f(x)$, 它在 $I$ 的某一稠密点集上点点取 到极大值,但是对于区间 $J \subseteq I$, 点集 $\{x ; f(x)=t, x \in J\}$ 若不是空集,则是可数集.

证 设 $A=[0,1), L$ 为 $A$ 中有理数的全体. 对于 $x, y \in A$, 定义 $\{x \pm y\}$ 为 $x$ 与 $y$ 的 和、差, 其中记号 $\{x\}$ 表示 $x$ 的小数部分. 对于每一 $\alpha \in A$. 记 $A_{\alpha}=\{\{\alpha+l\} ; l \in L\}$ ，显然， 对于 $\alpha_{1} \neq \alpha_{2}, A_{\alpha_{1}}$ 与 $A_{\alpha_{2}}$ 或重合, 或不相交. 从而必有集 $B$, 使

$$
A=\bigcup_{\alpha \in B} A_{\alpha} \quad\left(A_{\alpha} \cap A_{B}=\phi, \alpha \neq \beta\right) .
$$

易知, 集 $B$ 的势为连续统势 $c$. 事实上，

$$
A=\bigcup_{\alpha \in B} A_{\alpha}=\bigcup_{a \in B} \bigcup_{i=1}^{\infty}\left\{\alpha+l_{i}\right\}=\bigcup_{i=1}^{\infty} \bigcup_{a \in B}\left\{\alpha+l_{i}\right\},
$$

其中 $\left\{l_{i} ; i=1,2, \cdots\right\}=L$. 因为 $A$ 的势为 $c$, 所以必有某个 $i_{0}$, 使 $\bigcup_{\alpha \in B}\left\{\alpha+l_{i_{0}}\right\}$ 具有势 $c$, 即 $B$ 的势为 $C$.

于是,可建立集类 $\left\{A_{\alpha} ; \alpha \in B\right\}$ 和 $[0,1]$ 之间的一一对应. 记对应于 $A_{\alpha}$ 的数为 $\alpha$. 今定 义 $I$ 上的函数如下:

$$
f(x)= \begin{cases}\bar{\alpha}, & x \in A_{s}(\alpha \in B) \\ 0, & x=1\end{cases}
$$

本文 1977 年 9 月 27 日收到. 1979 年 2 月 19 日收到修改稿. 
我们证明函数 $f(x)$ 即满足定理要求.

由于 $L$ 在 $I$ 上稠密, 不难知道集 $A_{a}$ 均稠密于 $I$. 设对应于数 1 的集 $A_{\alpha}$ 不妨记为 $A_{1}$ 那么 函数 $f(x)$ 在稠密于 $I$ 的集 $A_{1}$ 上取到极大值 (Solomon Marcus 的命题是对广义极大值而言的).

当 $t \bar{\epsilon} I$ 时,集 $\{x ; f(x)=t, x \in J \subseteq I\}$ 为空集; 当 $t=\bar{a} \in I$ 时,集 $\{x ; f(x)=\bar{a}, x \in J \subseteq$ $I\}=A_{a} \cap J$ 仍为可数. 事实上, 若 $A_{\alpha} \cap J$ 为有限, 则 $A_{\alpha}$ 在 $J$ 中不椆密了. 此时, 我们也已证 明了函数 $f(x)$ 具有达布性质一取一切函数中间值. 不仅如此, 在 $[0,1]$ 中的任意区间里该 函数都具有达布性质. 因此, $f(x)$ 便是满足定理的达布函数.

上述定义的函数，显然处处间断、处处振幅为 1 ，处处近旁可取到函数一切中间值. 这样， 我们就顺便给出了一种构造处处间断的达布函数的简便方法.

由于 $\left\{A_{\alpha} ; \alpha \in B\right\}$ 与 $[0,1]$ 的一一对应方法至少有 $c$ 种, 因此有

推论处处间断的达布函数至少有 $c$ 个.

下面,我们构造一类非连续的达布函数满足 Marcus 命题要求.

定义 对任意 $[c, d] \subset[a, b]$, 集 $E \cap[c, d]$ 具有势 $c$, 则称 $E$ 在 $[a, b]$ 上 $c$-稠密.

引理 在区间 $[0,1]$ 上存在着两两不相交的 $c$ 个 $c$-稠密集.

证 由定理 1 之证明，我们得到了 $[0,1]$ 上的稠密的两两不相交的 $c$ 个集 $A_{\alpha}(\alpha \in B)$, 其中有一个是 $[0,1)$ 上的有理数集 $L$. 如果除去集 $L$, 那么集 $A_{\alpha}$ 中的点均不为二进位有理 点,于是集 $A_{a}$ 中的点均是二进位无理点. 设

$$
\begin{aligned}
A_{\alpha} & =\left\{x_{n}(\alpha) ; n=1,2, \cdots\right\}, \\
x_{n}(\alpha) & =0 . \xi_{1}^{(\alpha)}(\alpha) \xi_{2}^{(n)}(\alpha) \cdots \xi_{m}^{(n)}(\alpha) \cdots,
\end{aligned}
$$

其中 $\xi_{m}^{(n)}(\alpha)=0$ 或 1 , 且不会从某一数位后都为 0 或都为 1 .

将自然数集 $N$ 分解为可数个可数集之和:

$$
N=\bigcup_{n=1}^{\infty} N_{n}, \quad N_{n}=\left\{2^{n-1}(2 k-1) ; k=1,2, \cdots\right\} \text {. }
$$

简记数 $2^{n-1}(2 k-1)$ 为 $(n, k)$. 利用点 $x_{n}(\alpha)$ 在数轴上的位置构造如下点集

其中置:

$$
X_{n}(\alpha)=\left\{0 . \xi_{1}^{(n)}(\alpha) \xi_{2}^{(n)}(\alpha) \cdots \xi \sum_{(n, n)}^{(n)}(\alpha) \eta_{(n, n)+1} \cdots \eta_{(n, n)+p} \cdots\right\},
$$

(i) $\eta_{(n, n+(2 q-1))}=\xi_{(n, n)+q}^{(n)}(\alpha) \quad(q=1,2, \cdots)$;

(ii) $\eta_{(n, n+2 q)}=0$ 或 $1(q=1,2, \cdots)$, 这就决定了数集 $X_{n}(\alpha)$ 具有势 $c$;

(iii) 在剩余的未定义过的足码 $(n, n)+p$ 位置上使 $\eta_{(n, n)+p}=0$.

容易验证, 当 $\alpha \neq \beta$ 时, $X_{n}(\alpha) \cap X_{n}(\beta)=\phi$, 这是由于对 $\xi_{m}^{(n)}(\alpha)$ 与 $\xi_{m}^{(n)}(\beta)$ 来说总有 $m$ 使 其不同. 还有。当 $n \neq m$ 时，显然有 $X_{n}(\alpha) \cap X_{m}(\alpha)=\phi$, 这是由于足标 $(n, n+i)$ 和 $(m$, $m+i)(i=1,2, \cdots)$ 无相同的.

令

$$
\mathbf{Y}_{\alpha}=\bigcup_{n=1}^{\infty} X_{n}(\alpha) \quad(\alpha \in B)
$$

则有 $Y_{a} \cap Y_{B}=\phi(\alpha \neq \beta)$.

我们证明 $Y_{a}$ 是 $[0,1]$ 上的 $c$ 稠密集. 设 $[c, d] \subset[0,1]$, 取自然数 $N$, 使

$$
\frac{1}{2^{(N, N)}}<\frac{d-c}{3} \text {. }
$$


因为 $A_{x}$ 在 $[0,1]$ 上稠密, 所以集 $\left\{x_{(N, N)+p}(\alpha) ; p=1,2, \cdots\right\}$ 也在 $[0,1]$ 上稠密. 于是存 在 $i>(N, N)$, 使

$$
c+\frac{1}{3}(d-c)<x_{i}(\alpha)<d-\frac{1}{3}(d-c) .
$$

对于 $x \in X_{i}(\alpha)$ ，显然有

$$
\left|x-x_{i}(\alpha)\right| \leqslant \frac{1}{2^{i}}<\frac{1}{2^{(N, N)}}<\frac{d-c}{3},
$$

从而得到 $X_{i}(\alpha) \subset[c, d]$. $X_{i}(\alpha)$ 的势为 $c$, 所以 $Y_{\alpha} \cap[c, d]$ 的势也为 $c$.

定理 2 , 在区间 $I=[0,1]$ 上存在达布函数 $f(x)$, 它在 $I$ 的某一稠密点集上点点取到 极大值,同时对于每一个区间 $J \subseteq I$ 和 $t \in f(I)$, 集 $J \cap\{x ; f(x)=t\}$ 具有势 $c$.

证设 $\left\{Y_{\alpha}\right\}$ 是 $I$ 中两两不相交的 $c$ 个 $c-$ 稠密集. 使 $\left\{Y_{\alpha}\right\}$ 与 $I$ 建立一一对应. 设对 应于 $a \in I$ 的为 $Y_{\alpha \text { : }}$ 定义 $I$ 上的函数如下:

$$
f(x)= \begin{cases}\vec{\alpha}, & x \in Y_{\alpha}(\alpha \in B) ; \\ 0, & x \in I-\left\{Y_{\alpha} ; \alpha \in B\right\} .\end{cases}
$$

不难验证函数 $f(x)$ 即满足定理要求。

\title{
考文献
}

[1] Marqus, S., Reone de Math. Pures et Appl., Acad. R. P., R., 8 (1963), 2:267-271.

[2] Hobson, E. W., Theory of Functions of a Real Variable II, 1926, 412-421.

[3] I. U. 德林费尔德,普通数学分析教程补篇(中译本), 人民教育出版社, 1960 .

\section{DARBOUX FUNCTION OSCILLATING EVERYWHERE}

\author{
Wang Zu-yue（王祖樾）
}

\begin{abstract}
This paper has negated the problem raised by Solomon Marcus ${ }^{[1]}$ and pointed out the existence of some Darboux functions which satisfy the proposition of Solomon Marcus. The following are the conclusions:

Theorem 1. There exists a Darboux function at the interval $I=[0,1]$ that takes its maximal value in the dense set but for any subinterval $J \subseteq I$, the set $\{x ; f(x)=t, x \in J\}$ is either void or countable.

Theorem 2. There exists a Darboux function at the interval $I=[0,1]$ that takes its maximal value in the dense set; but for any subinterval $J \subseteq I$ and $t \in f(I)$, the set $J \cap\{x ; f(x)=t\}$ has a cardinal c.
\end{abstract}

